Errata

\title{
Notes on the Nonlinearly Elastic Boussinessq Problem
}

J. G. SIMMONDS and P. G. WARNE

Department of Applied Mathematics, University of Virginia, Charlottesville, VA 22903-2442, USA

Journal of Elasticity 34: 69-82, 1994

Eq. (2): replace the minus sign with a plus sign.

Eq. (3): the second line should read “... if incompressible”.

Eq. (15): replace the minus sign with a plus sign.

Eq. (17): place a minus sign on the left in front of $\delta \mathscr{P}$. 